\title{
Articulaciones entre marxismo y feminismo: ayer y hoy
}

\author{
María Cecilia Espasandín ${ }^{1}$ \\ https://orcid.org/0000-0003-4628-2499 \\ ${ }^{1}$ Universidad de la República, Facultad de Ciencias Sociales, Departamento de Trabajo Social, Montevideo, Uruguay
}

\begin{abstract}
Articulaciones entre marxismo y feminismo: ayer y hoy
Resumen: El artículo introduce los análisis de tres pensadoras feministas que abordan la relación entre producción y reproducción, desde la perspectiva del materialismo histórico. La articulación de la perspectiva feminista y la perspectiva marxista en las autoras tratadas posibilita visualizar las relaciones entre clase y género, y aún más significativamente, la intersección de distintas esferas de la vida social. El artículo es fruto de un proceso de investigación teórica en curso. Se estructura en cuatro partes: en las tres primeras, se expone someramente la contribución de cada una de las autoras y al final, se polemiza con algunas de sus interpretaciones a la luz de la lectura lukácsiana del materialismo histórico. El objetivo de la discusión es fortalecer los esfuerzos de articulación entre feminismo y marxismo, confiando en la potencialidad radical de este encuentro en el plano teórico y político.
\end{abstract}

Palabras clave: Feminismo. Marxismo. Teoría social.

\section{Articulações entre marxismo e feminismo: ontem e hoje}

Resumo: $\mathrm{O}$ artigo introduz as análises de três pensadoras feministas que abordam a relação entre produção e reprodução, na perspectiva do materialismo histórico. A articulação da perspectiva feminista e da perspectiva marxista nas autoras tratadas permite visualizar as relações entre classe e gênero, e ainda mais significativamente, a intersecção de diferentes esferas da vida social. O artigo é o resultado de um processo de pesquisa teórica. Está estruturado em quatro partes: nos três primeiros, a contribuição de cada uma das autoras é brevemente exposta e, no final, algumas de suas interpretações são polemizadas à luz da leitura lukacsiana do materialismo histórico. $\mathrm{O}$ objetivo da discussão é fortalecer os esforços de articulação entre feminismo e marxismo, confiando no potencial radical deste encontro no plano teórico e político.

Palavras-chave: Feminismo. Marxismo. Teoria social.

\section{Articulations between Marxism and Feminism: Yesterday and Today}

Abstract: This article introduces the analyzes of three feminist authors that approach the relation between production and reproduction, in the perspective of historical materialism. The articulation of the feminist and the Marxist perspectives in the authors used for this study, allows to visualize the relations between class and gender, and even more significantly, the intersection of different spheres of social life. The study is a theoretical research structured in four parts: in the first three, the contribution of each of the authors is briefly exposed and, at the end, some of their interpretations are discussed based on a Lukacsian perspective of historical materialism. The aim of the discussion is to strengthen the connection between feminism and Marxism, relying on the radical potential of this encounter at the theoretical and political level.

Keywords: Feminism. Marxism. Social theory.

Recibido en 15.02.2018. Aprobado en 25.05.2018. Revisado en 27.07.2018.

\section{(ब) $(\triangle$}

(C) El(Los) Autor(es). 2018 Acceso Abierto Esta obra está licenciada bajo los términos de la Licencia Creative Commons Atribución-NoComercial 4.0 Internacional (https://creativecommons.org/licenses/by-nc/4.0/deed.es), que permite copiar, distribuir y reproducir en cualquier medio, así como también adaptar, transformar y crear a partir de este material, desde que para fines no comerciales, y que usted fornezca el crédito debido a los autores y a la fuente, insiera un enlace para la Licencia Creative Commons e indique si fueron hechas alteraciones. 


\section{Introducción}

El artículo introduce los análisis de las feministas contemporáneas Anna Jónasdóttir y Valiere Bryson con el objetivo de ilustrar las tentativas recientes de articulación del feminismo con el marxismo. Ambas recogen la vocación radical del feminismo y la promesa socialista - que podemos encontrar antes en Shulamith Firestone. Con la acumulación de las reflexiones producidas en las últimas cuatro décadas (al menos), acerca de la relación entre marxismo y feminismo, Jónasdóttir y Bryson se posicionan a favor de una revitalización del diálogo. Apuestan a una relectura del materialismo histórico en interlocución con otras corrientes feministas. En alusión al tema que nos convoca en el presente número de Katálysis, la intersección de las dimensiones de clase, raza, género y sexualidad es objeto de un análisis específico por parte de Shulamith Firestone. Pero es la propia articulación de la perspectiva feminista y la perspectiva marxista en las tres autoras mencionadas que elucida las relaciones entre clase y género, y aún más significativamente, entre distintas dimensiones de la vida social: entre naturaleza y cultura (en Firestone); entre trabajo y amor (en Jónasdóttir); entre producción y reproducción (en Bryson). El artículo es fruto de un proceso de investigación en curso para la elaboración de la tesis doctoral, escrito a partir de la revisión y análisis de material bibliográfico. Se presentan las tres contribuciones y al final, se introduce una lectura crítica de las mismas, en base a presupuestos teóricos lukácsianos. El objetivo de la discusión es fortalecer los esfuerzos de articulación entre feminismo y marxismo, confiando en la potencialidad radical de este encuentro en el plano teórico y político.

\section{Shulamith Firestone: naturaleza y cultura.}

En 1971, la feminista norteamericana Shulamith Firestone publica La dialéctica del sexo: en defensa de la revolución feminista; un texto referente del feminismo radical emergente, en el que intenta desarrollar una interpretación materialista de la historia basada en el sexo mismo.

Firestone formula el feminismo como un proyecto radical, en el sentido de la definición marxista de 'radical'. Radical significa tomar las cosas por la raíz y, por lo tanto, deberemos ir a la raíz misma de la opresión. Según Firestone, este análisis ha sido insuficiente hasta el momento, bien por parte de la tradición feminista, bien por parte de la izquierda y del materialismo histórico. (Amorós, 2014, p. 884).

Bajo la influencia de El segundo sexo de Simone De Beauvoir y la tradición freudo-marxista de la Escuela de Frankfurt (Wilhelm Reich y Hebert Marcuse), Firestone considera que el antagonismo de las clases sexuales impregna todo el curso de la historia. Retoma de Marx y Engels su "[...] método de análisis a la vez dialéctico y materialista", pero les cuestiona que su interpretación estrictamente económica de la lucha de clases no es capaz de explicar la opresión de la mujer (Firestone, 1976, p. 11). Entiende que existe todo un sustrato sexual en la dialéctica histórica - que Engels apenas entrevió - y que no deriva directamente de la economía.

Firestone (1976, p. 17) apela al determinismo biológico para explicar las relaciones de poder entre las "clases sexuales", hombre/mujer. La diferenciación reproductiva natural entre los sexos condujo a la primera división sexual del trabajo, en tanto que las mujeres, subordinadas a su propia biología - partos, amantamientos, cuidados de pequeños - se convirtieron en dependientes de los varones para su propia supervivencia. Su hincapié en la diferencia biológica para explicar la dialéctica del sexo, da a lugar a interpretaciones esencialistas. Enfatiza los componentes más esencialistas de su teoría en un capítulo dedicado al análisis de la cultura, calificada de "masculina" y en oposición a "[...] la cultura de un modo femenino." (Firestone, 1976, p. 201)".

Si la diferencia biológica explicaría el antagonismo entre los sexos en el curso de la historia, la supresión de la base biológica sería la solución. A diferencia de la idealización de la esencia femenina nutricia que hará el feminismo cultural, Firestone considera que la mujer debe ser liberada de su carga reproductiva, por la cual ha sido oprimida a lo largo de la historia. "A pesar de todo, conceder que el desequilibrio sexual del poder tiene una base biológica, no supone arruinar nuestra causa." (Firestone, 1976, p. 19). Confiando en el desarrollo técnico puesto al servicio de la reproducción (control de la fertilidad, reproducción artificial, etc.), propone "[...] la destrucción de la tiranía de la familia biológica", y específicamente, "[...] una vuelta a la pansexualidad sin trabas" que reemplazaría a la hetero/homo/bi-sexualidad (Firestone, 1976, p. 20).

Su peculiar propuesta se inspira en la proposición freudo-marxista de abolición de la represión sexual que imbuye la generación del mayo del '68. También explicita una analogía con la tesis marxista: si la revolución socialista se dirige a la eliminación de la distinción de clases (y no solo a la eliminación de los privilegios de una clase), entonces la revolución feminista se dirige a la eliminación de la distinción misma de sexo (y no solo a la eliminación de los privilegios masculinos). 
Firestone articula la revolución feminista con la revolución socialista. Bajo el paralelismo con la revuelta obrera por el control de los medios de producción, plantea la revuelta de las mujeres hacia la confiscación del control de la reproducción. No se restringe a una propuesta de naturaleza sexual; se trata del control de la propiedad de sus cuerpos, del control de las instituciones sociales destinadas al alumbramiento y educación de los hijos, de la dependencia del hijo respecto a un grupo y no la madre. Su intención radical corre junto a una perspectiva de totalidad. "Necesitaremos una revolución sexual mucho más amplia que la socialista -y, por supuesto, que la incluya - para erradicar verdaderamente todos los sistemas clasistas." (Firestone, 1976, p. 22). Da prioridad a las reivindicaciones específicamente feministas, cuestionando a las "políticas" (feministas de izquierda) que subordinan estas demandas a "la Gran Lucha" contra el capitalismo (Firestone, 1976, p. 47). El feminismo radical surge como el correlato teórico de esta práctica del feminismo como práctica no subsidiaria (Amorós, 2014).

Dos aspectos de su teoría revolucionaria merecen especial atención por su diálogo con el marxismo. El primero es su confianza en el desarrollo técnico. Paradójicamente, aunque atribuya a la naturaleza biológica la determinación fundante de las relaciones sociales, coloca en la naturaleza social la posibilidad de transformarlas. Gracias a la actuación práctica de la humanidad sobre la naturaleza, a la que no se somete pasivamente sino que se enfrenta y asume el control sobre ella en beneficio propio, "[...] ya no podemos justificar el mantenimiento de un sistema discriminatorio de clases sexuales basándonos en su enraizamiento en la Naturaleza." (Firestone, 1976, p. 19).

Aquí aparece su proximidad - mediante su lectura de Simone De Beauvoir - con la reflexión ontológica-social de Marx. La naturaleza humana surge como auto-creadora, capaz de hacer retroceder "la barrera natural" (Marx, 2004, p. 624) para construir su propia historia. Pero su proximidad tiene un límite claro: para Marx, el retroceso de las barreras naturales no implica su eliminación; para Firestone, se trata de la supresión misma de la base biológica. Sobre esto, se discute en el último apartado.

Ligada a la concepción socio-histórica del ser humano, emerge la política como acción práctica de transformación. Bajo el leimotiv de "lo personal es político" - propio del feminismo radical (Amorós, 2014, p. 821) -, denuncia que las tentativas de cambiar la vida personal encuentran las resistencias del poder masculino. "El problema se hace político: si bien el hombre es cada vez más capaz de librarse de las circunstancias biológicas (que crearon su tiranía sobre mujeres y niños), tiene poco motivos para desear renunciar a ella." (Firestone, 1976, p. 19).

Así el desarrollo técnico no aparece como un progreso inevitable hacia la emancipación humana. Al contrario, para Firestone (1976, p. 20), "[...] las nuevas técnicas - especialmente el control de la fertilidad - pueden convertirse en un arma hostil, utilizada para reforzar este arraigado sistema de explotación." El antagonismo entre las clases - en Firestone,

El objetivo de la discusión es fortalecer los esfuerzos de articulación entre feminismo y marxismo, confiando en la potencialidad radical de este encuentro en el plano teórico y político. sexuales - bloquea las posibilidades emancipatorias del desarrollo de las fuerzas productivas. Al igual que en Marx, la técnica no es una fuerza ciega del progreso, sino que debe ser puesta - mediante una práctica consciente - al servicio del mismo.

Dos secciones de su obra merecen un último destaque. La intersección de las dimensiones de clase, raza, género y sexualidad es objeto de un análisis "muy perspicaz" por parte de Firestone (Amorós, 2014, p. 1247). En un capítulo titulado El racismo o el sexismo de la raza humana, examina cómo se entrecruzan las jerarquías entre hombre blanco y mujer negra, hombre negro y mujer blanca, entre mujeres de distinto color y clase, y lo mismo entre hombres. Penetra en el fenómeno de exaltación de la mujer negra como madre y esposa en la literatura y actitudes de la "comunidad negra militante" (Firestone, 1976, p. 147); una solidaridad intergenérica de la raza negra que acaba no propiciando la emancipación de las mujeres negras. Analiza los pactos entre los hombres blancos de izquierda y los hombres negros, raíces del "machismo", sufrido por las mujeres al interior de los propios movimientos disidentes - antirracistas, anticapitalistas (Firestone, 1976, p. 134). Para el análisis de la intersección entre racismo y sexismo, se vale de su experiencia en el movimiento en defensa de los derechos civiles de la década de 1960 - movimiento disidente que actuó como "catalizador" del "movimiento de liberación de la mujer", según Firestone (1976, p. 44). De hecho, el feminismo radical surge bajo la influencia de la lucha antirracista y retoma la crítica a una situación de opresión - esta vez de la mujer.

Pero es en un capítulo dedicado al "Amor" que Firestone despliega "sus dotes como analista extraordinariamente fina del poder." (Amorós, 2014, p. 1281). Para Firestone (1976, p. 159), "el amor, más quizás 
que la gestación de los hijos, es el baluarte de la opresión de las mujeres en la actualidad." Su concepción de que las mujeres "viven para el amor" (Firestone, 1976, p. 160) la aproxima a las formulaciones de la revolucionaria bolchevique Alexandra Kollontai, quien - contra las resistencias de su tiempo - cuestionaba los rasgos psicológicos que caracterizaban a la mujer, relegada al papel de amar o ser amada. "Luciendo su vena más heterodoxa Kollontai critica explícitamente la postura marxista que mantiene que los problemas de amor son problemas de superestructura y que se solucionarían cuando cambie la base económica de la sociedad." (de Miguel, 2014, p. 2844).

Según Firestone, no hay un elemento intrínsecamente destructivo en el proceso amoroso. Puede haber un genuino "éxtasis amoroso", o enriquecimiento entre dos iguales, pero no suele ser frecuente; "[...] por cada breve período de enriquecimiento, encontramos diez experiencias amorosas destructivas." (Firestone, 1976, p. 162). Para comprender "[...] cómo actúa este fenómeno que llamamos 'amor' [...]", Firestone (1976, p. 161) profundiza en el terreno psicológico de las relaciones de poder, recurriendo al material clínico de Theodor Reik, ejemplos cotidianos y de la literatura. Identifica una idealización romántica en el enamoramiento masculino a causa de que "[...] el hombre necesita idealizar a una mujer sobre todas las demás, para así justificar su descenso a una clase inferior." (Firestone, 1976, p. 165). En cambio, la mujer sabe que esta idealización no es auténtica, pero busca la aprobación masculina. Esta falsa idealización es responsable de la faceta destructiva del amor y se debe al contexto político de desigualdad entre sexos. Pero el proceso mismo amoroso puede tener una idealización que signifique una captación más profunda, no falsa, del otro. En definitiva, "“...] sugiero que el amor es en esencia un fenómeno mucho más simple y que se complica, corrompe u obstruye por medio de un desequilibrio de poder." (Firestone, 1976, p. 163).

Firestone (1976, p. 185) encuentra en el amor romántico "[...] el amor corrompido por su contexto de poder - el sistema de clases sexuales [...]." Al ahondar en La cultura del romance amoroso - en el siguiente capítulo así titulado - trata de cómo el romanticismo se ha desarrollado para asentar la supremacía masculina, agudizándose a medida que la mujer se libera de los constreñimientos biológicos que la hacen clase sexual dependiente. "El romanticismo no es más que un instrumento cultural del poder masculino, cuya finalidad es mantener a las mujeres en la ignorancia de su condición." (Firestone, 1976, p. 186).

La feminista radical articula su crítica teórica al romanticismo al plano político de la revolución sexual. Identificando en el erotismo uno de los pilares del amor romántico, propone eliminarlo como tal, preguntándose: "¿Por qué toda la alegría y excitación ha sido concentrada y unificada en una parcela estrecha y difícil de alcanzar de la experiencia humana, dejando baldío todo lo demás?” (Firestone, 1976, p. 196). La eliminación del erotismo tal como lo conocemos sería sustituido por una redifusión del goce sexual.

\section{Anna Jónasdóttir: trabajo y amor.}

Es significativa la irradiación de La dialéctica del sexo. En defensa de la revolución feminista de Shulamith Firestone al interior del feminismo. A pesar de su alejamiento precoz del movimiento radical (Amorós, 2014) y que el libro "[...] ha sido despachado con demasiada premura por los marxistas [...]" (Hartmann, 1981, p. 10), su difusión es notoria. En su influyente artículo Un matrimonio mal avenido: hacia una unión más progresiva entre marxismo y feminismo, Hartmann (1981, p. 10) sostiene acerca del libro de Firestone:

Su capítulo sobre el amor era y sigue siendo fundamental para comprender esto. No es sólo una 'ideología machista' que los marxistas pueden afrontar (una mera cuestión de actitudes), sino una exposición de las consecuencias subjetivas del poder del hombre sobre la mujer, de lo que se siente al vivir en un patriarcado ${ }^{2}$.

Si nos centramos en la crítica de Firestone al amor, su influjo puede observarse en textos inmediatamente posteriores, siendo un ejemplo las teóricas del llamado "trabajo emocional" (Molina, 2014, p. 2478) 3 .

Según Amorós (2014, p. 1298), también “[...] en la tradición de la concepción firestoniana del amor se sitúa la sugerente elaboración teórica de la feminista nórdica Jónasdóttir." De origen más reciente, se trata de un análisis sobre "el poder del amor" para explicar la persistencia de la dominación masculina en las sociedades formalmente igualitarias (Jónasdóttir, 2011, p. 247). Para Molina (2014, p. 2462), quien reconstruye los debates del feminismo socialista y sus dificultades para analizar la opresión sexual con herramientas teóricas marxistas: "Desde la perspectiva socialista europea, Anna Jónasdóttir forzó el paso definitivo para tratar la sexualidad en estos términos."

Jónasdóttir explicita su diálogo con el marxismo. Es "[...] a través de una aplicación crítica y reconstructiva del método de Marx [...]”, que Jónasdóttir (2011, p. 248) arriba al amor "[...] como una 
capacidad humana creativa-productiva - y explotable - de importancia comparable a la del trabajo." Específicamente, identifica el amor sexual como "[...] un elemento fundamental en el proceso de producción de personas." (Jónasdóttir, 2011, p. 257).

En otras palabras, las relaciones sociosexuales (alias relaciones de género), las prácticas amorosas, así como la lucha y el control sobre los usos del poder del amor en el proceso de producción-reproducción de personas comprenden una dimensión social particular, identificable como tal para el estudio de las sociedades y del cambio social. (Jónasdóttir, 2011, p. 248).

Tomando distancia de la teoría feminista posestructuralista dominante durante los años 1980 y 1990, Jónasdóttir (2011, p. 252) retoma el "proyecto teórico" de articulación entre el feminismo y el marxismo de los años 1970, que suponía problematizar "el sexo" sin subsumirlo bajo "la clase" y analizar la desigualdad de sexo mediante el método de Marx. No obstante la diversidad de teorías feministas inspiradas en el materialismo histórico, la mayor parte "[...] cayó en un estancamiento durante los años ochenta bajo la presión del posestructuralismo y la multifacética 'guerra' contra el marxismo." (Jónasdóttir, 2011, p. 255). Citando un conjunto de obras contemporáneas, Jónasdóttir (2011, p. 255) concluye que, "[...] un renovado y considerablemente fuerte interés en la obra de Marx puede observarse en todo el mundo, tanto entre las teóricas feministas como en otros autores." A partir de su reinterpretación del materialismo histórico, propone una reconstrucción feminista de la perspectiva marxiana de la producción, centrada, ya no en el trabajo, sino en el amor. Con este "giro", Jónasdóttir (2011, p. 249) apuesta ir "[...] más allá de las nociones tajantes o excluyentes que aún son un gran obstáculo para los debates del materialismo versus la teoría de la cultura."

Desde su perspectiva - centrada en la sexualidad política - considera que las recientes investigaciones inspiradas en el materialismo histórico no se dirigen al estudio de las causas de la dominación masculina sobre las mujeres. Ella entiende que es el poder de los hombres el que requiere ser explicado - y no la opresión o subordinación de las mujeres. En las sociedades capitalistas contemporáneas (formalmente igualitarias), no es la dependencia económica de las mujeres respecto a los hombres, ni es la división sexual desigual del trabajo entre los sexos, lo que explica la continuidad del patriarcado (aunque reconoce su importancia).

Lo que es crucial es la posesividad de los hombres con respecto a las mujeres; es decir, el derecho que los hombres reclaman para tener acceso a las mujeres. En la práctica, los "derechos" de los hombres para apropiarse de los recursos sociosexuales de las mujeres, especialmente de su capacidad para el amor, continúa siendo un patrón predominante. (Jónasdóttir, 2011, p. 255).

La autora establece una analogía entre algunos componentes de la teoría marxiana (la producción de los medios de subsistencia, las clases sociales y la organización del trabajo) y los componentes de su propia teoría (la producción de la vida, las relaciones de género y la organización del amor). La explotación del amor brindado libremente bajo el "[...] patriarcado formalmente igualitario [...]” (Jónasdóttir, 2011, p. 260) es lo que en Marx, la explotación del trabajo asalariado vendido libremente bajo el capitalismo. Retirando del término explotación cualquier dimensión moral, reivindica el estudio de las formas normales de uso-disfrute del amor - y no solo las formas abusivas de explotación sexual. El patriarcado hoy se sostiene, fundamentalmente, por las relaciones sexuales libres que se establecen entre hombres y mujeres, en las cuales éstas son explotadas por aquellos, sobre todo en su capacidad para el amor. Como afirma: “[...] decir que las capacidades humanas para el amor y para el trabajo pueden explotarse es como afirmar que éstas son fuentes vivientes de energía que pueden ser liberadas." (Jónasdóttir, 2011, p. 259).

Amor y trabajo son prácticas humanas creativas - práctica humano-sensorial, en los términos de Marx - y como tales, portan "capacidades creadoras de mundos", es decir, capacidades para cambiar las circunstancias y a uno mismo (Jónasdóttir, 2011, p. 249). Pero difieren entre sí en un aspecto. El ser humano realiza la práctica del trabajo bajo la conciencia de un propósito, bajo la previa ideación del resultado de su actividad - como ilustra Marx (2002, p. 216) en su famoso ejemplo donde distingue el "peor maestro albañil de la mejor abeja." En cambio, la práctica humana del amor, dice Jónasdóttir (2011, p. 264), no se orienta por una previa ideación del resultado.

El paradigma del arquitecto y la abeja no sirve para captar lo que distingue al amor como una actividad humana opuesta al comportamiento biológicamente determinado. Para que el amor sea una actividad genuinamente humana su practicante actúa no para hacer que el objeto del amor coincida con una 'idea ya existente' sino más bien para permitir que el 'objeto' del amor confirme su propia capacidad para 'crearse' o 'moldearse' a sí mismo(a) y para sus propias metas. 
Se podría polemizar con esta lectura de Jónasdóttir, a la luz de la interpretación lukácsiana de Marx. Se trata esto en el último apartado.

No obstante trabajo y amor se distingan en un aspecto, considera que están íntimamente relacionados. El proceso específico de crear personas-vidas y existencia sociosexual se encuentra entrecruzado con la producción de medios de subsistencia (la dimensión económica de la sociedad). Jónasdóttir (2011, p. 264) concluye, entonces, que: "[...] debería esperarse que los cambios en las condiciones del amor y del trabajo respectivamente tuvieran un efecto recíproco."

Jónasdóttir examina la explotación de la capacidad de amor al exponer las contradicciones internas del amor en la sociedad contemporánea. Los componentes principales del amor son el cuidado y el éxtasis erótico. Ambos están en una continua contradicción debido a la manera en que las relaciones amorosas heterosexuales se institucionalizan. "Cuando mujeres y hombres (legalmente libres e iguales) se conocen como sexos, las condiciones sistémicas en que suceden esos encuentros no son igualitarias." (Jónasdóttir, 2011, p. 265). Bajo el modo de producción de personas en la sociedad contemporánea, juega un papel central la autoafirmación sexual-erótica. El acceso a experiencias de éxtasis erótico aparece como una condición previa para la dignidad de la persona. Esta clase de poder sexual requiere del cuidado amoroso, para que sea creado y cargado de valor.

Típicamente, en la forma predominante de dichos encuentros 'hombre-mujer', la 'mujer' es 'forzada' a comprometerse al cuidado amoroso para que el 'hombre' pueda ser capaz de vivir-experimentar el éxtasis. No es igualmente legítimo que la 'mujer' practique el éxtasis como una persona sexual con dirección y seguridad propias, quien, al hacerlo, necesita del cuidado del hombre (iy éste es un punto de vital importancia!). (Jónasdóttir, 2011, p. 265).

La “[...] posición sistémica de los hombres los presiona hacia un ilimitado deseo de éxtasis [...]" como medio para reafirmar su poder sexual; mientras que "[...] práctica del cuidado amoroso en sus relaciones con las mujeres tiende a ser concebida como una serie de cargas y limitaciones [...]" (Jónasdóttir, 2011, p. 266). Suceden entonces, que bajo las condiciones sistémicas del patriarcado, los hombres acceden al empoderamiento que les otorga explotar la capacidad de amor de las mujeres.

Molina (2014, p. 2482) reconstruye el análisis de Jónasdóttir en los siguientes términos:

La organización de la sexualidad en nuestras sociedades en la que los hombres ejercen la autoridad que les da el 'poder del amor' (explotando la necesidad que la mujer tiene de amar ser amada) es el vector de opresión más importante en las mujeres de hoy, desplazando el trabajo y las determinaciones económicas de su protagonismo inicial.

\section{Valerie Bryson: producción y reproducción.}

Una de las autoras que Jónasdóttir identifica en la senda actual de investigaciones feministas inspiradas en la obra de Marx es la feminista británica Valerie Bryson, junto a la cual ha trabajado (Jónasdóttir, Bryson, \& Jones, 2011). En su artículo Marxism and feminism: can the 'unhappy marriage' be save? Bryson (2006) se propone extender el diálogo entre marxismo y feminismo 4 .

La feminista británica construye su análisis en base a la concepción materialista de la historia. No secunda las interpretaciones que asignan un determinismo económico al materialismo histórico - y que aún hoy debaten al respecto. Se asienta en la famosa formulación de Marx (1973, p. 408) de que los seres humanos hacen su propia historia pero en condiciones que no eligen, sino que heredan del pasado.

No obstante, acuerda con la crítica feminista que atribuye una base y un sesgo masculinos a la teoría marxista, pues esta "[...] ha estado basada en una perspectiva limitada que en gran parte ignora importantes áreas de la experiencia humana." (Bryson, 2006, p. 10). Alude, en términos generales, a la esfera reproductiva. Propone una articulación teórica entre marxismo y feminismo, a favor de una noción ampliada de (re)producción, que incluya el trabajo socialmente necesario realizado desproporcionadamente por las mujeres. Entiende por (re)producción: "Aquellas actividades humanas (físicas y emocionales) que están más o menos directamente vinculadas con la reproducción generacional y el mantenimiento de la población y el cuidado de aquellos imposibilitados de cuidarse a sí mismo.” (Bryson, 2006, p. 13).

Distingue su concepto (re)producción de la concepción clásica de la reproducción. El término reproducción ha tenido un uso indiscriminado con distintos significados atribuibles, como: proceso biológico de procreación; reproducción de la fuerza de trabajo; reproducción del circuito de la economía misma; o reproducción 
de las relaciones sociales. Las actividades reproductivas comprendidas bajo su concepto incluyen, no solo la procreación biológica, sino además cocinar, limpiar, el cuidado de los niños, personas mayores y personas con discapacidad o enfermos, y la satisfacción de necesidades emocionales y sexuales. Pueden ser desarrolladas individualmente en el hogar, o vistas como responsabilidades comunales o estatales, o remuneradas como parte de la economía del dinero (y por lo tanto también productivas en el sentido estricto). En todos los casos están mayoritariamente asociadas a las mujeres.

La reflexión de Bryson acerca de la producción y la (re)producción se inspira en un acervo de debates producidos al interior del feminismo socialista alrededor de los años sesenta y setenta. Al igual que el feminismo anterior, Bryson (2005) retoma las contribuciones de Marx y Engels - sobre todo de El origen de la familia, la propiedad privada y el Estado de Engels (1892) - para el estudio de la esfera reproductiva. Reconoce las elaboraciones de la socialdemócrata alemana Clara Zetkin y su destacado liderazgo al frente del movimiento de mujeres durante la Segunda Internacional Socialista. Y en particular, revisita las propuestas familiares revolucionarias de Alexandra Kollontai en el marco de la revolución bolchevique - vale recordar que, para Kollontai, la colectivización del trabajo doméstico y del cuidado y la liberación de la sexualidad respecto a las ideas de propiedad facilitaban el desarrollo del tipo de moral colectiva necesaria para que floreciese la economía socialista. Bryson recorre las obras clásicas del marxismo respecto a la situación de la mujer (que merecen una atención aparte y que por razones de espacio no podemos reproducir) y concluye que generalmente han oscurecido la subordinación femenina, pues " [...] asumieron que la opresión culminaría en una sociedad socialista, sin explorar la posibilidad de que esta podría tener su propia dinámica, basada en condiciones de la vida reproductiva." (Bryson, 2010, p. 4). La conclusión de Bryson se basa en las críticas que el feminismo socialista dirigió al marxismo en los años sesenta y setenta - y que pueden encontrarse de manera sintética en el artículo ya citado de Heidi Hartmann (1981). En particular, se funda en las críticas feministas originadas en el marco del debate sobre el trabajo doméstico (naciente en los años sesenta) y del debate sobre el trabajo productivo e improductivo (de los años setenta), cuya exponente destacada es Maria Rosa Dalla Costa (Galcerán, 2006).

Desde estas fuentes, Bryson cuestiona que la teoría del valor de Marx no tomó en consideración el trabajo de producción de la propia fuerza de trabajo, o trabajo de reproducción y de cuidados. Entiende que la perspectiva materialista marxista recentrada en la mujer implica una reconceptualización del término producción. Si bien en la teoría marxista este término es utilizado, a veces, para referirse al trabajo dirigido a la satisfacción de las necesidades humanas, normalmente no ha sido interpretado para incluir el trabajo de reproducción de la especie, el cual ha sido tratado como natural y sin lugar en la historia. Según Bryson (2006, p. 12) la teoría marxista asocia el trabajo productivo con el trabajo asalariado que produce plusvalor porque está "afectado por los valores capitalistas hegemónicos".

Voy a sugerir que el sesgo masculino de muchos teóricos marxistas dominantes ha sido reforzado por una ecuación subconsciente de 'productivo' con 'importante', y esto ayuda a explicar por qué han mostrado poco interés en analizar la cambiante naturaleza del trabajo no remunerado que es en gran parte hecho por mujeres y su compleja relación con la economía del dinero. (Bryson, 2006, p. 12).

La reflexión sobre producción y reproducción que hoy vuelve a recolocar Bryson corre de la mano de diversas exponentes. Con una tonalidad menos radical - pero conviviendo con un horizonte socialista - la reflexión de Bryson apunta a identificar las mediaciones entre producción y reproducción. Se distancia de Shulamith Firestone y su tentativa - "simplista" (Bryson, 2010, p. 9) - de reconstruir la concepción materialista de Marx sustituyendo producción por reproducción e identificando la organización sexualreproductiva como la clave de la opresión social. Para Bryson, el desafío del análisis de la producción y (re)producción está en explorar los límites cambiantes y las formas en que cada uno media y se ve afectado por los cambios en el otro. En este sentido, la feminista británica propone reexplorar los modos en que las fuerzas del mercado se extienden a áreas de la vida que el capitalismo había tratado usualmente como privadas - es el caso de las nuevas tecnologías reproductivas que facilitan la explotación de las capacidades reproductivas de las mujeres. Invita a estudiar los cambios en las condiciones de (re)producción y sus efectos en la producción; y hasta dónde la reducción del tiempo necesario para las actividades reproductivas habría permitido combinar más fácilmente trabajo remunerado y no remunerado para las mujeres y haberles facilitado el acceso a puestos de jerarquía. Bryson relativiza estos efectos, dada la persistente inequidad entre mujeres y hombres en el trabajo o en el hogar. La economía capitalista y la maximización del lucro limitan prerrequisitos fundamentales para la equidad sexual. "Esto significa que sería extraordinariamente dificultoso terminar con la opresión patriarcal sin cambios económicos radicales, incluyendo un salto hacia mayores responsabilidades colectivas y hacia una producción para el uso o las necesidades más que para el lucro." (Bryson, 2006, p. 16). 


\section{Contribuciones lukácsianas para una articulación entre feminismo y marxismo}

El propósito de este apartado es hacer dialogar los aportes de las tres autoras con las contribuciones de György Lukács (2012), en la búsqueda de una aproximación a la obra marxiana que desvele más nítidamente las posibilidades de articulación entre feminismo y socialismo. Para eso, se retoman globalmente los ejes de la exposición: producción y reproducción; trabajo y amor; naturaleza y cultura.

La concepción materialista de la historia considera que el trabajo - la esfera económica de la sociedad - es fundante de todas las determinaciones del ser social. Esto no quiere decir que todo fenómeno social pueda deducirse del trabajo, o reducirse a este. Sin embargo, es una lectura recurrente y puede observarse en la bibliografía feminista, como por ejemplo, en Shulamith Firestone. Firestone critica a Marx y Engels su interpretación estrictamente económica de la lucha de clases. Aun cuando no se asigne un determinismo económico al materialismo histórico y se lo interprete como una teoría dialéctica entre condiciones heredadas y práctica humana - como vimos en Bryson - se desprende que Marx subestimó los aspectos de la reproducción (biológica, cultural, afectiva-emocional, etc.). Su énfasis en la producción no le permitió captar la particularidad de otras prácticas humanas, como la práctica del amor - afirma - a la que no se puede comprender mediante el marxiano "paradigma del arquitecto y la abeja” (Jónasdóttir, 2011, p. 263).

Profundizando en los fundamentos ontológicos del ser social, el filósofo húngaro György Lukács (2012) hace otra lectura de la producción y reproducción en Marx - que aquí apenas se introduce.

Tal como afirma Jónasdóttir, Marx encuentra en el trabajo la actividad propiamente humana, que lo distingue de los animales. Desde sus orígenes, el ser humano es capaz de modificar conscientemente la naturaleza para producir los medios indispensables de vida. A diferencia de los animales, no responde con una adaptación reactiva al ambiente, sino que crea objetos útiles para satisfacer sus necesidades (de alimento, vivienda, abrigo, etc.). El trabajo y los productos del trabajo introducen en la vida necesidades siempre nuevas y hasta ahí desconocidas y, con ellas, nuevos modos de satisfacerlas; "[...] volviendo la reproducción de la vida siempre más variada y compleja, distanciándola cada vez más de la reproducción biológica." (Lukács, 2012, p. 269). El ser social se hace "siempre más netamente y puramente social" haciendo "retroceder la barrera natural" - es decir, desarrollando capacidades para convertir los procesos regidos por la naturaleza (causalidades naturales) en causalidades puestas - y la propia diferenciación biológica entre los miembros del grupo humano "[...] asume en sí momentos de socialidad siempre más numerosos" (Lukács, 2012, p. 138). Desde esta concepción, retroceso no implica destrucción de la naturaleza, como en Firestone.

En su forma más simple, el trabajo es una relación entre ser humano y naturaleza: el ser humano transforma la naturaleza orientado por una posición teleológica (una conciencia que pone fines) cuyo fin es transformar objetos naturales en valores de uso. En las formas evolucionadas de la praxis social (política, arte, religión, ciencia, etc.), los actos teleológicos no buscan realizar una transformación de la naturaleza, sino que se proponen ejercer influencia sobre la conciencia de otros seres humanos. En este sentido, el trabajo se distingue de otras formas de actividad - como sostiene Jónasdóttir - pero - a diferencia de ella -, para Lukács, el trabajo es modelo de toda praxis social, pues contiene la forma originaria de posiciones teleológicas que en el curso de la historia tendrán un desarrollo más complejo 5 .

Más allá de la distinción entre tipos de actos teleológicos, lo que resulta importante rescatar es la lectura marxiana-lukácsiana de la dialéctica entre libertad y determinación en la esfera del trabajo - y de ahí en las otras formas de praxis social, también en la esfera afectiva. Así, Lukács critica la idea de la producción - o llámese la economía - como mundo objetivo puro, y contrapuesto al mundo de pura subjetividad, porque también "lo económico" es "la síntesis legal de aquellos actos teleológicos que cada uno de nosotros efectúa ininterrumpidamente.” (Lukács, 2012, p. 245).

Así entendido el papel de la conciencia en el proceso de trabajo - y de ahí, en otras formas de praxis lleva a reconocer que el ser humano, en sus actos, sus ideas y también en sus sentimientos, siempre está dando una "respuesta a un dilema" (Lukács, 2012, p. 127) que la sociedad determinada en la que vive le coloca. No hay en Lukács una interpretación del individuo como simple producto de su medio, pero tampoco la idea de una sustancia atemporal de la individualidad humana. Lukács constata la influencia del ser social "[...] sobre las más íntimas, las más personales formas de pensamiento, de sensibilidad, de acción y de reacción de cada individuo humano.” (Lukács, 2012, p. 260).

Una lectura lukácsiana invita a comprender el amor desde los fundamentos ontológico-sociales y en sus expresiones histórico-concretas: ¿por qué el amor se presenta hoy y aquí así? Claro que requiere de un estudio concreto sobre esta esfera específica. Pero, a diferencia de las autoras tratadas, entiendo que la especificidad de esta esfera es pasible de ser aprehendida en su conexión orgánica con la praxis del trabajo - y sin una inversión de su jerarquía ontológica. 


\section{Consideraciones finales}

La exposición teórica de las tres autoras fue sumamente introductoria pero sirve para constatar la reactualización de debates entre marxismo y feminismo. El somero recorrido permite desempolvar el pensamiento marxista al interior del feminismo y redescubrir contribuciones sepultadas por la posterior reacción ofensiva. Re-transitar las críticas al marxismo formuladas por corrientes feministas - mismo por aquellas que derivan en las antípodas del materialismo histórico, como puede ser el feminismo cultural - posibilita relanzar el diálogo sobre nuevas bases; en otras palabras, contribuye a elucidar los límites a superar.

La desigualdad de género es la preocupación central en las tres feministas tratadas y es entendida como una problemática no subsidiaria a otras - ni secundaria como lucha -, ni superable en los marcos de la igualdad formal. Para abordarla, hacen una relectura del materialismo histórico. Si nos centramos en los ejes de la exposición, las autoras tratan la intersección entre naturaleza y cultura, trabajo y amor, producción y reproducción, como dimensiones de la vida social que solo pueden ser comprendidas desde una perspectiva de totalidad. Aquí se considera que la lectura marxiana de Lukács esclarece el entramado de relaciones subjetivas y objetivas implicadas.

\section{Referencias}

Amorós, C. (2014). ‘La dialéctica del sexo’ de Shulamith Firestone: modulaciones feministas del freudo-marxismo. In C. Amorós, \& A. de Miguel (Eds.), Teoría feminista: de la Ilustración a la globalización [versión Kindle] (Vol. 2, Cap. 3). Madrid: Minerva.

Bryson, V. (2006). Marxism and feminism: can the 'unhappy marriage'be saved? Recuperado de http://www.tandfonline.com/doi/abs/ 10.1080/1356931032000167454

Bryson, V. (2010). Production and Reproduction. Recuperado de https://caringlabor.wordpress.com/2010/07/28/valerie-brysonproduction-and-reproduction/

de Miguel, A. (2014). La articulación del feminismo y el socialismo: el conflicto clase-género. In: C. Amorós, \& A. de Miguel (Eds.) Teoría feminista: de la Ilustración a la globalización [versión Kindle] (Vol. 1, Cap. 7). Madrid: Minerva.

Firestone, S. (1971). La dialéctica del sexo: en defensa de la revolución feminista. Barcelona: Kayrós.

Galcerán, M. (2006). Producción y reproducción en Marx. In Laboratorio Feminista (Ed.), Transformaciones del trabajo desde una perspectiva feminista: producción, reproducción, deseo, consumo (pp. 13-26). Madrid: Tierradenadie.

Hartmann, H. (1981). Un matrimonio mal avenido: hacia una unión más progresiva entre marxismo y feminismo. Recuperado de http:/ /www.fcampalans.cat/uploads/publicacions/pdf/88.pdf

Jónasdóttir, A. G. (2011). ¿Qué clase de poder es “el poder del amor”? Sociológica, 26(74), 247-273.

Jónasdóttir, A. G., Bryson, V., \& Jones, K. (Eds.). (2011). Sexuality, gender and power: intersectional and transnational perspective. Londres: Routledge.

Lukács, G. (2012). Ontologia dell'essere sociale (Vol. 3). Milán: PGRECO.

Marx, K. (1973). El dieciocho Brumario de Luis Bonaparte. In K. Marx, \& F. Engels. Obras Escogidas (Vol. 1, pp. 408-498). Moscú: Progreso.

Marx, K. (2002). El capital. Libro primero (Vol. 1). Buenos Aires: Siglo Veintiuno.

Marx, K. (2004). El capital. Libro primero (Vol. 2). Buenos Aires: Siglo Veintiuno.

Molina, C. (2014). El feminismo socialista estadounidense desde la "Nueva Izquierda": las teorías del sistema dual (capitalismo + patriarcado). In C. Amorós, \& A. de Miguel (Eds.), Teoría feminista: de la Ilustración a la globalización [versión Kindle] (Vol. 2, Cap. 5). Madrid: Minerva.

Osborne, R. (2014). Debates en torno al feminismo cultural. In C. Amorós, \& A. de Miguel (Eds.), Teoría feminista: de la Ilustración a la globalización [versión Kindle] (Vol. 2, Cap. 7). Madrid: Minerva.

\section{Notas}

1 De hecho, una deriva del feminismo radical es el feminismo cultural, que se despliega en la década de 1980 y exacerba el determinismo biológico para explicar la dualidad hombre/mujer (Osborne, 2014). Atribuyendo cualidades femeninas y masculinas a causas de naturaleza sexual, el feminismo cultural “[...] ya no contiene ninguna crítica específica al capitalismo.” (Amorós, 2014, p. 854).

2 No cabe aquí desarrollar las reflexiones de Hartmann, pero su propuesta de interpretación de un sistema dual actuante-capitalismo y patriarcado reforzándose mutuamente - fue catalizador y, "[...] en torno a su metáfora del 'matrimonio desgraciado’ se desarrolló gran parte de la teoría feminista socialista americana de los años 80.” (Molina, 2014, p. 2440).

3 Las socialistas Anna Ferguson y Nancy Folbre difunden el término "producción sexo-afectiva"; que Sandra Lee Bartky retoma para analizar la apropiación masculina de las capacidades emocionales femeninas y los efectos alienantes (Molina, 2014, p. 2317). Allí puede leerse la idea delineada por Firestone (1976, p. 160) de una cultura que “[...] se alimenta de la energía emocional de las mujeres sin reciprocidad.” 
4 Contraria a tildar este diálogo de filosófica o "políticamente anticuado", Bryson (2006, p. 2) expone tres argumentos para fundamentar que marxismo y feminismo no están ultrapasados: 1) en el plano político, la existencia de movimientos políticos anticapitalistas (marxistas) y actividades feministas; 2) en el plano teórico, la continuidad de los estudios feministas y el resurgimiento del interés en el marxismo; 3) la realidad del capitalismo de libre mercado y las relaciones de género como formas históricas, transitorias.

5 No es posible desarrollar aquí todas las consecuencias que se extraen del proceso de trabajo y que lo hacen modelo de toda praxis. Vale destacar que se desprende el núcleo ontológico de la libertad. La libertad es una voluntad, en última instancia, de transformar la realidad (o preservarla) y se origina en el proceso de trabajo, donde toda posición tiene un carácter alternativo, un momento de decisión. Se trata de decisiones alternativas concretas, en el sentido que existe un determinado campo de fuerzas para las decisiones, en el cual actúan fuerzas naturales y sociales. Esto implica que, en las formas de praxis más complejas, los actos teleológicos que tienen por objeto suscitar en los seres humanos determinadas decisiones entre alternativas, actúan en un material que, de por sí, espontáneamente tiende a decidir entre alternativas.

\section{María Cecilia Espasandín}

macecilia.espasandin@cienciassociales.edu.uy

Magíster en Trabajo Social por la Universidade Federal do Rio de Janeiro (UFRJ)

Profesora Adjunta del Departamento de Trabajo Social de la Facultad de Ciencias Sociales de la Universidad de la República (UDELAR)

\section{UDELAR}

Facultad de Ciencias Sociales

Constituyente, 1502

Montevideo - Uruguay

CP: 11.200

\section{Agencia financiadora}

No se aplica.

\section{Contribuciones de los autores}

No se aplica.

\section{Consentimiento para publicación \\ No se aplica.}

\section{Conflicto de intereses}

No hay conflicto de intereses.

Aprobación por Comisión de Ética y consentimiento para participación

No se aplica. 